\title{
Nonsteroidal Anti-inflammatory Drugs-sparing Effect of Symptomatic Slow-acting Drugs for Osteoarthritis in Knee Osteoarthritis Patients
}

\author{
Soo-Kyung Cho ${ }^{1}$, Hyoungyoung Kim ${ }^{1}$, Ha-Rim Park ${ }^{1}$, Wooseok Choi $^{2}$, Seongmi Choi $^{2}$, Sun-Young Jung ${ }^{3}$, \\ Eun Jin Jang ${ }^{4}$, Yoon-Kyoung Sung ${ }^{1}$ \\ ${ }^{1}$ Department of Rheumatology, Hanyang University Hospital for Rheumatic Diseases, Seoul, ${ }^{2}$ Department of Statistics, Kyungpook National \\ University, Daegu, ${ }^{3}$ College of Pharmacy, Chung-Ang University, Seoul, ${ }^{4}$ Department of Information Statistics, Andong National University, \\ Andong, Korea
}

Objective. To evaluate the nonsteroidal anti-inflammatory drugs (NSAID)-sparing effect of symptomatic slow-acting drugs for osteoarthritis (SYSADOA) in knee osteoarthritis (OA) patients. Methods. A retrospective study was conducted on a cohort of knee OA patients who visited a single academic referral hospital from 2013 to 2014. Among all patients, NSAID users in their first visit were extracted and divided into SYSADOA users and SYSADOA non-users. All patients were observed from their first visit with knee OA to their last visit, NSAID discontinuation, or the date of data collection, July 2017 (mean observational periods: 369.1 days). To evaluate the NSAID-sparing effect of SYSADOA, Cox regression analysis was performed after adjusting for confounding factors. Results. Patients for this study $(n=212)$ were divided into SYSADOA users $(n=57)$ and SYSADOA non-users $(n=155)$. The mean age $(68.8$ vs. 66.6 years old, $p=0.31)$ and the number of comorbidities $(p=0.73)$ were comparable between the two groups. The SYSADOA users showed higher Kellgren-Lawrence (KL) grade $(66.7 \%$ of patients with more than KL grade 3) than SYSADOA non-users ( $42.6 \%$ of patients with more than $K L$ grade 3$)(p=0.02)$. In treatment, the frequency of intra-articular injection was higher in the SYSADOA user group than the SYSADOA non-user group (33.3\% vs. 9.0\%, $\mathrm{p}<0.01$ ). In Cox regression analysis, SYSADOA use contributed to NSAID discontinuation in knee OA patients (hazard ratio $2.97,95 \%$ confidential interval $1.42 \sim 6.22$ ). Conclusion. This real-world analysis demonstrated that SYSADOA use combined with NSAIDs had a significant effect on NSAID discontinuation in patients with knee OA. (J Rheum Dis 2019;26:179-185)

Key Words. Osteoarthritis, Symptomatic slow-acting drugs for osteoarthritis, Nonsteroidal anti-inflammatory drugs

\section{INTRODUCTION}

Osteoarthritis $(\mathrm{OA})$ is a common form of arthritis and most frequently affects the knee, causing joint pain, tenderness, limitation of movement, and a reduction in quality of life, imposing a social and economic burden [1,2]. Roughly $25 \%$ of adults aged 55 years or older experience knee pain on most days, and most of these adults have osteoarthritis [3]. In Korea, the prevalence of radiologic OA and symptomatic OA was reported as $37.3 \%$ and $24.2 \%$, respectively, in a community survey of elderly residents
[4]. In a recent report using the Fifth Korean National Health and Nutrition Examination Survey-V (2010 2012), the number of knee OA patients with symptoms and radiological findings in males and females was $4.5 \%$ and $19 \%$, respectively [5].

Several therapies are available to reduce OA-associated symptoms. The most commonly used treatment options are focused on pain relief and improvement of joint function, and include non-steroidal anti-inflammatory drugs (NSAIDs), analgesics (acetaminophen or opioids), and symptomatic slow-acting drugs for OA (SYSADOA).

\footnotetext{
Received : December 26, 2018, Revised : (1st) February 8, 2019, (2nd) March 13, 2019, Accepted : March 13, 2019

Corresponding to : Yoon-Kyoung Sung (10http://orcid.org/0000-0001-6691-8939

Department of Rheumatology, Hanyang University Hospital for Rheumatic Diseases, 222 Wangsimni-ro, Seongdong-gu, Seoul 04763, Korea. E-mail : sungyk@hanyang.ac.kr
}

Copyright (c) 2019 by The Korean College of Rheumatology. All rights reserved.

This is an Open Access article, which permits unrestricted non-commerical use, distribution, and reproduction in any medium, provided the original work is properly cited. 
These drugs are recommended in many knee OA guidelines [6-10]. NSAIDs are mainstays for knee OA treatment, but have dose-related risks including adverse gastrointestinal and cardiovascular events [11,12]. Expecting to reduce the use of NSAIDs, various clinical trials have been performed to compare the effectiveness and safety of SYSADOA and NSAID treatment [13-18].

The use of SYSADOA in knee OA patients at $43.4 \%$ in Korea [19] was comparable to other countries (27.5\% $54.0 \%$ ) for US [20] and its regular use with $37.3 \%$ in Korea was $46.6 \%$ in a study from Spain [21]. However, individual drug selection differed in different countries. In the US and European countries, compounds such as glucosamine or chondroitin are frequently used, while in Korea new herbal medications are frequently prescribed [19]. The use of glucosamine or chondroitin has dramatically decreased, since they were excluded from Korean reimbursement guidelines based on the Glucosamine/ Chondroitin Arthritis Intervention Trial (GAIT) study showing that glucosamine/chondroitin did not reduce pain effectively [14], although they were previously widely used by the general population and not limited to knee OA patients [22]. Therefore, we aimed to evaluate the NSAID-sparing effect of SYSADOA including new herbal medications in patients with knee OA.

\section{MATERIALS AND METHODS}

\section{Data source and study participants}

The data source was hospital medical records including inpatients and outpatients from a single academic referral hospital. A retrospective cohort of patients with knee OA confirmed by knee X-ray, who first visited one university hospital from 2013 to 2014, was conducted.

\section{Study design and data collection}

Comprehensive chart reviews for all patients were undertaken by well-trained health professionals; data included demographic data such as age, gender, and clinical data such as comorbidities, the result of a knee X-ray, treatment including NSAIDs, gastrointestinal protecting agents, and intra-articular injection. Well-known SYSADOAs such as diacerein, avocado soybean unsaponifiables, and chondroitin were included in this study, but glucosamine was excluded since it has not been reimbursed by Health Insurance and Review Assessment since 2011. Instead, herbal drugs combining herbal extracts including JOINS [23] (a mixed extract of three herbs: Clematis mandshurica, Prunella vulgaris, and Trichosanthes kirilowii), LAYLA [24] (an ethanol extract prepared from 12 plant sources: Chaenomelis fructus, Achyranthis radix, Acanthopanax cortex, Cinnamomi cortex, Gentianae macrophyllae radix, Clematidis radix, Angelica gigantis radix, Cnidii rhizoma, Gastrodiae rhizoma, Carthami flos, Saposhnikoviae radix, and Dipsaci radix), and SHINBARO [25] (a purified extract from a mixture of 6 oriental herbs: Ledebouriellae radix, Achyranthis radix, Acanthopanacis cortex, Cibotii rhizoma, Glycine semen, and Eucommiae cortex) were included.

\section{Outcome assessment}

We assessed the cases which did not use any NSAIDs and the reasons for discontinuation upon chart review. The reasons for discontinuation of NSAIDs were categorized as: adverse events, good effect, ineffectiveness, or unknown. Switching to a different NSAID was considered as continuation of NSAIDs. Observations started from the first visit with knee OA and ended with the last visit, NSAIDs discontinuation, or the date of data collection, July 2017, whichever came first.

\section{Ethical considerations}

This study was approved by the regional Institutional Review Board (IRB no. HYUH 2016-06-008-002). Informed consent was not required because data was stripped of identifying information and collected retrospectively.

\section{Statistical analysis}

Descriptive analysis with mean and standard deviation (SD) [26] and chi-square test or independent t-test for comparison of two groups was used. The NSAID-sparing effect of SYSADOA was estimated by comparing the percentage of patients who stopped NSAIDs during the observational periods. Cox regression analysis was performed to evaluate this after adjusting for confounding factors. Confounding factors of the p-value $<0.3$ in unadjusted analysis were considered. Medications such as NSAIDs, SYSADOAs, analgesics, and corticosteroids during the observational periods were used by time dependent variables. All analyses were performed using the SAS version 9 software package (SAS Institute, Cary, NC, USA). 


\section{RESULTS}

\section{Demographic and clinical characteristics of patients with knee osteoarthritis}

Of all the patients enrolled in this study $(\mathrm{n}=768)$, NSAID users in their first visit $(n=212)$ were extracted and divided into SYSADOA users $(\mathrm{n}=57)$ and SYSADOA non-users $(n=155)$. The baseline study population characteristics are summarized in Table 1. Mean age, proportion of females, and the prevalence of chronic comorbidities such as hypertension, diabetes mellitus, and chronic kidney disease were similar between the two groups. However, SYSADOA users were treated in the department of rehabilitation more frequently ( $47.4 \%$ vs. $11.0 \%, \mathrm{p}<0.01)$ and by orthopedic surgery less frequently $(10.5 \%$ vs. $38.1 \%, p=0.74)$. The frequency of Kellgren-Lawrence (KL) grade 3 or 4 in the SYSADOA users was higher than in the SYSADOA non-users ( $63.2 \%$ vs. $41.3 \%$ in $\mathrm{KL}$ grade $3,3.5 \%$ vs. $1.3 \%$ in $\mathrm{KL}$ grade $4, \mathrm{p}=0.02$ ).

\section{Treatment patterns of knee OA patients according to SYSADOA use}

SYSADOA users used more analgesics $(71.9 \%$ vs. $58.7 \%, \mathrm{p}=0.08$ ) but less corticosteroids ( $7.0 \%$ vs. $11.6 \%$, $\mathrm{p}=0.33$ ) compared to patients using NSAIDs without SYSADOA, though the difference was not statistically significant. There was no difference between the two groups in the use of gastroprotective agents. The frequency of intra-articular injection was higher in the SYSADOA users than in the non-users $(33.3 \%$ vs. $9.0 \%$, $\mathrm{p}<0.01)$. Intra-articular injection with hyaluronic acid was more frequent in SYSADOA users than in the non-users $(26.3 \%$ vs. $5.8 \%, \mathrm{p}<0.01)$. Intra-articular injection with corticosteroids was more frequent in SYSADOA users than in non-users, but the difference was not statistically significant ( $7.0 \%$ vs. $3.2 \%, p=0.25$ ) (Table 2).

Table 1. Baseline and clinical characteristics of study population

\begin{tabular}{|c|c|c|c|c|}
\hline Variable & Total $(n=212)$ & $\begin{array}{l}\text { SYSADOA users } \\
(\mathrm{n}=57)\end{array}$ & $\begin{array}{l}\text { SYSADOA non-users } \\
\qquad(\mathrm{n}=155)\end{array}$ & $\mathrm{p}$-value \\
\hline Age (yr) & $67.9 \pm 7.7$ & $68.8 \pm 8.0$ & $66.6 \pm 7.6$ & 0.31 \\
\hline Sex (female) & $184(86.8)$ & $48(84.2)$ & $136(87.7)$ & 0.50 \\
\hline Outpatient visits & 199 (93.9) & $56(98.3)$ & $143(92.3)$ & 0.19 \\
\hline Rheumatology & $123(58.0)$ & $32(56.1)$ & $91(58.7)$ & $<0.01$ \\
\hline Orthopedic surgery & $65(30.7)$ & $6(10.5)$ & $59(38.1)$ & 0.74 \\
\hline Rehabilitation & $44(20.8)$ & $27(47.4)$ & $17(11.0)$ & $<0.01$ \\
\hline Cardiology & $16(7.6)$ & $2(3.5)$ & $14(9.0)$ & 0.25 \\
\hline Gastroenterology & $3(1.4)$ & 0 & $3(1.9)$ & 0.57 \\
\hline General surgery & $1(0.5)$ & 0 & $1(0.7)$ & 1.00 \\
\hline \multicolumn{5}{|c|}{ Previous history of chronic diseases } \\
\hline Hypertension & 67 (32.9) & $16(28.1)$ & $51(32.9)$ & 0.50 \\
\hline Diabetes mellitus & $27(13.5)$ & $6(10.5)$ & $21(13.5)$ & 0.56 \\
\hline Chronic kidney disease* & $58(27.4)$ & $16(28.1)$ & $42(27.1)$ & 0.89 \\
\hline Gastrointestinal disease & $5(2.4)$ & 0 & $5(3.2)$ & 0.33 \\
\hline \multicolumn{5}{|l|}{ Number of comorbidities ${ }^{\dagger}$} \\
\hline 0 & 199 (93.9) & $53(93.0)$ & $146(94.2)$ & 0.73 \\
\hline 1 & $9(4.3)$ & $3(5.3)$ & $6(3.9)$ & \\
\hline$\geq 2$ & $4(1.9)$ & $1(1.8)$ & $3(1.9)$ & \\
\hline \multicolumn{5}{|c|}{ Knee $X$-ray $K L$ grade at first visit } \\
\hline KL grade 1 & $42(19.8)$ & $10(17.5)$ & $32(20.7)$ & 0.02 \\
\hline$K L$ grade 2 & 57 (26.9) & $9(15.8)$ & $48(31.0)$ & \\
\hline $\mathrm{KL}$ grade 3 & $100(47.2)$ & $36(63.2)$ & $64(41.3)$ & \\
\hline$K L$ grade 4 & $4(1.9)$ & $2(3.5)$ & $2(1.3)$ & \\
\hline
\end{tabular}

Values are presented as mean \pm standard deviation or number $(\%)$. SYSADOA: symptomatic slow-acting drugs for osteoarthritis, KL grade: Kellgren-Lawrence grade. ${ }^{*}$ Chronic kidney diseases were defined by $<60 \mathrm{~mL} / \mathrm{min} / 1.73 \mathrm{~m}^{2}$ of glomerular filtration rate. ${ }^{\dagger}$ Comorbidities were identified using the Charlson comorbidity index. 
Soo-Kyung Cho et al.

Table 2. Comparison of treatment patterns in SYSADOA users and SYSADOA non-users during the observation period

\begin{tabular}{|c|c|c|c|c|}
\hline Variable & Total $(n=212)$ & $\begin{array}{l}\text { SYSADOA users } \\
\qquad(n=57)\end{array}$ & $\begin{array}{l}\text { SYSADOA non-users } \\
\qquad(\mathrm{n}=155)\end{array}$ & p-value \\
\hline Analgesics & $132(62.3)$ & $41(71.9)$ & $91(58.7)$ & 0.08 \\
\hline Corticosteroids & $22(10.4)$ & $4(7.0)$ & $13(11.6)$ & 0.33 \\
\hline GI protect med & $170(80.2)$ & $47(82.5)$ & $123(79.4)$ & 0.62 \\
\hline Proton pump inhibitor & $50(23.6)$ & $10(17.5)$ & $40(25.8)$ & 0.21 \\
\hline $\mathrm{H} 2$ blocker & $11(5.2)$ & $3(5.3)$ & $8(5.2)$ & 1.00 \\
\hline Rebamipide & $1(0.5)$ & $0(0)$ & $1(0.6)$ & 1.00 \\
\hline Sucralfate & $40(18.9)$ & $13(22.8)$ & $27(17.4)$ & 0.37 \\
\hline Artemisia asiatica & $7(3.3)$ & $2(3.5)$ & $5(3.2)$ & 1.00 \\
\hline Teprenone & $11(5.2)$ & $3(5.3)$ & $8(5.2)$ & 1.00 \\
\hline Others & $123(58)$ & $35(61.4)$ & $88(56.8)$ & 0.54 \\
\hline Intra-articular injection & $33(15.6)$ & $19(33.3)$ & $14(9.0)$ & $<0.01$ \\
\hline Corticosteroids injection & $9(4.2)$ & $4(7.0)$ & $5(3.2)$ & 0.25 \\
\hline Hyaluronic acid injection & $24(11.3)$ & $15(26.3)$ & $9(5.8)$ & $<0.01$ \\
\hline Total knee replacement & $16(7.5)$ & $3(5.3)$ & $13(8.4)$ & 0.45 \\
\hline
\end{tabular}

Values are presented as number (\%). SYSADOA: symptomatic slow acting drugs for osteoarthritis, Gl: gastrointestinal.

Table 3. Factors influencing on NSAID discontinuation

\begin{tabular}{|c|c|c|c|c|}
\hline \multirow{2}{*}{ Variable } & \multicolumn{2}{|c|}{ Unadjusted } & \multicolumn{2}{|c|}{ Adjusted } \\
\hline & $\mathrm{HR}(95 \% \mathrm{Cl})$ & $p$-value & $\mathrm{HR}(95 \% \mathrm{Cl})$ & $\mathrm{p}$-value \\
\hline Age & $1.00(0.97 \sim 1.04)$ & 0.80 & $0.97(0.92 \sim 1.01)$ & 0.14 \\
\hline Sex & $0.96(0.34 \sim 2.68)$ & 0.93 & $0.94(0.33 \sim 2.67)$ & 0.91 \\
\hline \multicolumn{5}{|l|}{ Comorbidities } \\
\hline Gastrointestinal disease & $2.26(0.54 \sim 9.39)$ & 0.26 & $4.74(1.06 \sim 21.27)$ & 0.04 \\
\hline Cardiovascular disease & $0.92(0.50 \sim 1.72)$ & 0.80 & & \\
\hline Diabetes mellitus & $0.56(0.17 \sim 1.80)$ & 0.33 & & \\
\hline Chronic kidney disease* & $1.59(0.74 \sim 3.42)$ & 0.24 & $1.22(0.35 \sim 4.31)$ & 0.76 \\
\hline Number of comorbidities & $0.77(0.35 \sim 1.69)$ & 0.51 & & \\
\hline $\mathrm{KL}$ grade & $1.64(1.15 \sim 2.35)$ & 0.01 & $1.39(0.92 \sim 2.11)$ & 0.12 \\
\hline IAI with corticosteroid & $0.63(0.15 \sim 2.59)$ & 0.52 & & \\
\hline IAI with hyaluronic acid & $1.36(0.58 \sim 3.23)$ & 0.48 & & \\
\hline TKR & $4.13(2.12 \sim 8.04)$ & $<0.01$ & $6.21(2.66 \sim 14.49)$ & $<0.01$ \\
\hline \multicolumn{5}{|l|}{ Medication $^{\dagger}$} \\
\hline SYSADOA & $1.90(1.02 \sim 3.54)$ & 0.04 & $2.97(1.42 \sim 6.22)$ & $<0.01$ \\
\hline Analgesics & $2.16(1.18 \sim 3.94)$ & 0.01 & $1.43(0.75 \sim 2.71)$ & 0.28 \\
\hline Corticosteroids & $0.33(0.05 \sim 2.40)$ & 0.27 & $0.32(0.04 \sim 2.36)$ & 0.26 \\
\hline
\end{tabular}

NSAIDs: nonsteroidal anti-inflammatory drugs, HR: hazard ratio, $\mathrm{Cl}$ : confidence interval, KL grade: Kellgren-Lawrence grade, IAI: intra-articular injection, TKR: total knee replacement, SYSADOA: symptomatic slow-acting drugs for osteoarthritis. ${ }^{*}$ Chronic kidney diseases were defined by $<60 \mathrm{~mL} / \mathrm{min} / 1.73 \mathrm{~m}^{2}$ of glomerular filtration rate. ${ }^{\dagger}$ Medication during the observational period was used by time dependent variables.

\section{Impact of SYSADOA use on the discontinuation of NSAIDs}

The mean ( \pm SD) observational period was 369.1 $( \pm 423.0)$ days. Of the total patient group $(n=212), 44$ patients discontinued NSAIDs during follow-up. Their treatment duration with NSAIDs was mean $310.0( \pm 325.6)$ days (median, Q2, Q3 [159.0, 35.0, 556.5] days). The survival curve of NSAIDs was presented in Supplementary Figure 1. In terms of their reasons for discontinuation, 25 patients discontinued due to good effects, 9 patients due to adverse events, and 4 patients due to ineffectiveness. Six patients did not identify their reasons for discontinua- 
tion of NSAIDs. The frequency of NSAID discontinuation during follow-up was similar in the SYSADOA user group $(n=13)$ and the non-user group $(n=31)$ at $22.8 \%$ vs. $20.0 \%$, respectively $(\mathrm{p}=0.66)$. When the reason for NSAID discontinuation was limited to "good effect," the prevalence in each group was not different $(12.3 \%$ in the SYSADOA user group and $11.6 \%$ in the SYSADOA non-user group, $\mathrm{p}=0.89$ ).

In the Cox proportional hazard model adjusted for age, sex, comorbidities, KL grade in knee X-ray, and total knee replacement (TKR), SYSADOA use was associated with a higher probability of NSAID discontinuation (hazard ratio, HR 2.97, 95\% confidence interval, CI 1.42 6.22) (Table 3). Comorbidity with gastrointestinal disease (HR $4.74,95 \%$ CI 1.06 21.27) was also associated with the discontinuation of NSAIDs. In addition, TKR (HR 6.21, 95\% CI 2.66 14.49) was associated with the discontinuation of NSAIDs. When we evaluated the impact of each type of SYSADOA on the discontinuation of NSAIDs, it was found to be different for each drug (Supplementary Table 1).

\section{DISCUSSION}

In this retrospective cohort study, we found that SYSADOA use combined with NSAIDs had a significant effect on NSAID discontinuation in patients with knee OA.

To date, several clinical trials have shown the pain relief effects of SYSADOA to be similar to the effects of NSAIDs, and it is anticipated that these findings can lead to a reduction in NSAID use. Of the several types of SYSADOA, glucosamine and chondroitin compounds widely used around the world were extracted from animal products that appear to be capable of increasing proteoglycan synthesis in articular cartilage [27]. Previously, the randomized controlled study (RCT) named the GAIT study showed that glucosamine and chondroitin sulfate alone did not reduce pain effectively, but suggested that a combination of glucosamine and chondroitin sulfate might be effective in the subgroup of patients with moderate-to-severe knee pain [14]. A recent RCT study showed that the use of chondroitin sulfate and glucosamine had a comparable effect to celecoxib [15] and the observational study showed that crystalline glucosamine sulfate decreased the use of NSAIDs [18]. Another SYSADOA, diacerein, a purified compound with an anthraquinonic structure, inhibits interleukin (IL)- $1 \beta$, thereby retarding pathological processes initiated in $\mathrm{OA}$ [28]. Diacerin was found to be as effective as piroxicam in reducing knee OA pain [17], and when combined with diclofenac significantly decreased pain compared to treatment with diclofenac alone [16].

On the other hand, there is scarce evidence for the effectiveness and safety of new herbal SYSADOAs such as JOINS, LAYLA, and SYNBARO, which are frequently used in Korean patients with OA, although there are several reports regarding their biological effects. JOINS could be beneficial for pain relief with anti-inflammatory effects related to its inhibition of $\mathrm{IL}^{-1} \beta$, prostaglandin E2, and tumor necrosis factor (TNF)- $\alpha$ [23]. LAYLA showed anti-inflammatory activity via the suppression of pro-inflammatory cytokine expression, cartilage-protective effects via regulation of tissue inhibitors of metalloproteinases and matrix metalloproteinase production [29], and showed pain relief effects similar to celecoxib in the treatment of symptomatic knee OA [24]. SYNBARO downregulates inflammatory mediators such as inducible nitric oxide synthase, cyclooxygenase-2, and TNF- $\alpha$ and increases the pain threshold in the peripheral system [25]. Our study provides important evidence for the beneficial effects of these SYSADOA types on the reduction of NSAID use.

In our results, gastrointestinal disease closely related with adverse events of NSAIDs also showed a significant association with the discontinuation of NSAIDs. In addition, TKR was also strongly associated with the discontinuation of NSAIDs. When we analyzed the effects of SYSADOAs on the discontinuation of NSAIDs due to good effectiveness, the association of gastrointestinal diseases with the discontinuation of NSAIDs disappeared, while the association with TKR remained (data not shown). These results suggest that NSAID discontinuation might result from the development of adverse events or the pain relief following TKR. However, we did not present this finding here because the small number of NSAID discontinuations due to good effects decreased its statistical power.

This study has some limitations. First, the number of patients treated with SYSADOA was small because we extracted early OA patients who started with a combination of SYSADOAs and NSAIDs. This study design allowed us to avoid several confounding factors such as disease duration or previous drug usage, but the size of the study population was decreased substantially. Second, possible indication biases or unmeasured confounding factors re- 
main, although we adjusted for major comorbidities that could have affected drug selection and disease severity using KL grade. Third, we could not confirm whether the effects of SYSADOAs on the discontinuation of NSAIDs resulted from real pain relief or placebo effects. Further RCTs will be helpful to clarify the effectiveness of SYSADOAs for reduction of NSAID use.

This observational study evaluated the effectiveness of SYSADOAs including new herbal drugs which are frequently used only in Korea. Our findings indicate that these drugs have the potential to be good treatment options with NSAID-sparing effects. Further prospective RCTs will be needed to confirm this.

\section{CONCLUSION}

This real-world analysis demonstrated that SYSADOA use combined with NSAIDs had a significant effect on NSAID discontinuation in patients with knee OA.

\section{ACKNOWLEDGMENTS}

This research was supported by a grant of the Korea Health Technology R\&D Project through the Korea Health Industry Development Institute (KHIDI), funded by the Ministry of Health \& Welfare, Republic of Korea (grant number: $\mathrm{HC} 15 \mathrm{C} 3388$ ).

\section{CONFLICT OF INTEREST}

No potential conflict of interest relevant to this article was reported.

\section{SUPPLEMENTARY DATA}

Supplementary data can be found with this article online at https://doi.org/10.4078/jrd.2019.26.3.179.

\section{REFERENCES}

1. Woolf AD, Pfleger B. Burden of major musculoskeletal conditions. Bull World Health Organ 2003;81:646-56.

2. Murray CJ, Vos T, Lozano R, Naghavi M, Flaxman AD, Michaud C, et al. Disability-adjusted life years (DALYs) for 291 diseases and injuries in 21 regions, 1990-2010: a systematic analysis for the Global Burden of Disease study 2010. Lancet 2012;380:2197-223.

3. Peat G, McCarney R, Croft P. Knee pain and osteoarthritis in older adults: a review of community burden and current use of primary health care. Ann Rheum Dis 2001;60:91-7.
4. Kim I, Kim HA, Seo YI, Song YW, Jeong JY, Kim DH. The prevalence of knee osteoarthritis in elderly community residents in Korea. J Korean Med Sci 2010;25:293-8.

5. Park JH, Hong JY, Han K, Suh SW, Park SY, Yang JH, et al. Prevalence of symptomatic hip, knee, and spine osteoarthritis nationwide health survey analysis of an elderly Korean population. Medicine (Baltimore) 2017;96:e6372.

6. Jevsevar DS. Treatment of osteoarthritis of the knee: evidence-based guideline, 2nd edition. J Am Acad Orthop Surg 2013;21:571-6.

7. Hochberg MC, Altman RD, April KT, Benkhalti M, Guyatt G, McGowan J, et al. American College of Rheumatology 2012 recommendations for the use of nonpharmacologic and pharmacologic therapies in osteoarthritis of the hand, hip, and knee. Arthritis Care Res (Hoboken) 2012;64: 465-74.

8. National Clinical Guideline Centre (UK). Osteoarthritis: care and management in adults. London: National Institute for Health and Care Excellence (UK), 2014.

9. McAlindon TE, Bannuru RR, Sullivan MC, Arden NK, Berenbaum F, Bierma-Zeinstra SM, et al. OARSI guidelines for the non-surgical management of knee osteoarthritis. Osteoarthritis Cartilage 2014;22:363-88.

10. Jordan KM, Arden NK, Doherty M, Bannwarth B, Bijlsma JW, Dieppe P, et al.; Standing Committee for International Clinical Studies Including Therapeutic Trials ESCISIT. EULAR recommendations 2003: an evidence based approach to the management of knee osteoarthritis: report of a task force of the Standing Committee for International Clinical Studies Including Therapeutic Trials (ESCISIT). Ann Rheum Dis 2003;62:1145-55.

11. Wolfe MM, Lichtenstein DR, Singh G. Gastrointestinal toxicity of nonsteroidal antiinflammatory drugs. N Engl J Med 1999;340:1888-99.

12. Coxib and traditional NSAID Trialists' (CNT) Collaboration, Bhala N, Emberson J, Merhi A, Abramson S, Arber N, et al. Vascular and upper gastrointestinal effects of non-steroidal anti-inflammatory drugs: meta-analyses of individual participant data from randomised trials. Lancet 2013;382: 769-79.

13. Morreale P, Manopulo R, Galati M, Boccanera L, Saponati G, Bocchi L. Comparison of the antiinflammatory efficacy of chondroitin sulfate and diclofenac sodium in patients with knee osteoarthritis. J Rheumatol 1996;23:1385-91.

14. Clegg DO, Reda DJ, Harris CL, Klein MA, O'Dell JR, Hooper $\mathrm{MM}$, et al. Glucosamine, chondroitin sulfate, and the two in combination for painful knee osteoarthritis. N Engl J Med 2006;354:795-808.

15. Hochberg MC, Martel-Pelletier J, Monfort J, Möller I, Castillo JR, Arden N, et al. Combined chondroitin sulfate and glucosamine for painful knee osteoarthritis: a multicentre, randomised, double-blind, non-inferiority trial versus celecoxib. Ann Rheum Dis 2016;75:37-44.

16. Singh K, Sharma R, Rai J. Diacerein as adjuvant to diclofenac sodium in osteoarthritis knee. Int J Rheum Dis 2012; 15:69-77.

17. Louthrenoo W, Nilganuwong S, Aksaranugraha S, Asavatanabodee P, Saengnipanthkul S; Thai Study Group. The efficacy, safety and carry-over effect of diacerein in the treatment of painful knee osteoarthritis: a randomised, double-blind, NSAID-controlled study. Osteoarthritis Cartilage 
2007; 15:605-14

18. Rovati LC, Girolami F, D'Amato M, Giacovelli G. Effects of glucosamine sulfate on the use of rescue non-steroidal anti-inflammatory drugs in knee osteoarthritis: results from the Pharmaco-Epidemiology of GonArthroSis (PEGASus) study. Semin Arthritis Rheum 2016;45(4 Suppl):S34-41.

19. Park HR, Cho SK, Im SG, Jung SY, Kim D, Jang EJ, et al. Treatment patterns of knee osteoarthritis patients in Korea. Korean J Intern Med 2018 Mar 21 [Epub]. DOI:10.3904/ kjim.2017.304.

20. Lapane KL, Liu SH, Dubé CE, Driban JB, McAlindon TE, Eaton CB. Factors associated with the use of hyaluronic acid and corticosteroid injections among patients with radiographically confirmed knee osteoarthritis: a retrospective data analysis. Clin Ther 2017;39:347-58.

21. Wilson N, Sanchez-Riera L, Morros R, Diez-Perez A, Javaid MK, Cooper C, et al. Drug utilization in patients with OA: a population-based study. Rheumatology (Oxford) 2015; 54:860-7.

22. Seo HJ, Sung YK, Choi CB, Lee EB, Cheong C, Kim SY, et al. Prevalence and factors affecting glucosamine use in Korea: a survey-based study. Rheumatol Int 2013;33:1627-31.

23. Hartog A, Hougee S, Faber J, Sanders A, Zuurman C, Smit $\mathrm{HF}$, et al. The multicomponent phytopharmaceutical SKI306X inhibits in vitro cartilage degradation and the production of inflammatory mediators. Phytomedicine 2008; 15:313-20.

24. Yoo WH, Yoo HG, Park SH, Baek HJ, Lee YJ, Shim SC, et al.
Efficacy and safety of PG201 (Layla ${ }^{\circledR}$ ) and celecoxib in the treatment of symptomatic knee osteoarthritis: a double-blinded, randomized, multi-center, active drug comparative, parallel-group, non-inferiority, phase III study. Rheumatol Int 2014;34:1369-78.

25. Lee SY, Kwon HK, Lee SM. SHINBARO, a new herbal medicine with multifunctional mechanism for joint disease: first therapeutic application for the treatment of osteoarthritis. Arch Pharm Res 2011;34:1773-7.

26. Cui J, Stahl EA, Saevarsdottir S, Miceli C, Diogo D, Trynka $\mathrm{G}$, et al. Genome-wide association study and gene expression analysis identifies CD84 as a predictor of response to etanercept therapy in rheumatoid arthritis. PLoS Genet 2013;9:e1003394.

27. Bassleer C, Rovati L, Franchimont P. Stimulation of proteoglycan production by glucosamine sulfate in chondrocytes isolated from human osteoarthritic articular cartilage in vitro. Osteoarthritis Cartilage 1998;6:427-34.

28. Pelletier JP, Mineau F, Fernandes JC, Duval N, Martel-Pelletier J. Diacerhein and rhein reduce the interleukin 1beta stimulated inducible nitric oxide synthesis level and activity while stimulating cyclooxygenase-2 synthesis in human osteoarthritic chondrocytes. J Rheumatol 1998; 25:2417-24.

29. Shin SS, Jin M, Jung HJ, Kim B, Jeon H, Choi JJ, et al. Suppressive effects of PG201, an ethanol extract from herbs, on collagen-induced arthritis in mice. Rheumatology (Oxford) 2003;42:665-72. 